\title{
Idiopathic intracranial hypertension in a child being treated for systemic onset juvenile idiopathic arthritis
}

\author{
J Wanigasinghe ${ }^{1}$, M N Lucas ${ }^{2}$, S Jayanetthi ${ }^{3}$, S P Lamabadusuriya ${ }^{4}$ \\ Sri Lanka Journal of Child Health, 2010; 39: 115-116
}

(Key words: Idiopathic intracranial hypertension, systemic onset juvenile idiopathic arthritis)

\section{Introduction}

Idiopathic intracranial hypertension (IIH) is rare in children. Its association with diseases or therapies specific to childhood have been described ${ }^{1}$. An association between systemic onset juvenile idiopathic arthritis (SOJIA) and IIH has not been described before; however several drugs used for its treatment have been implicated. This case is reported due to the rarity of IIH in children.

\section{Case report}

A 5-year old boy presented with severe headache, vomiting and fever. He has been diagnosed with resistant SOJIA, requiring treatment with several anti-inflammatory and disease-modifying drugs. He was on high-dose oral steroids, hydroxychloroquine, ibuprofen, calcium lactate, vitamin A \& D, famotidine, gaviscon, vitamin-B-complex and folicacid on admission.

Severe headache made him curl up. Vomiting, though present, was not restricted to early morning. Examination revealed no signs of meningeal irritation. Fundoscopy showed right sided papilloedema (nasal margin-lower segment). Left fundus was normal. No other cranial nerves were involved. His visual acuity and visual field testing by confrontation was normal. Perimetry was abandoned due to severe neck pain.

Investigations revealed leucocytosis $\left(30 \times 10^{9} / \mathrm{L}\right)$ with neutrophil predominance. ESR was $53 \mathrm{~mm} / 1 \mathrm{st}$ hour, platelet count $235 \times 10^{9} / \mathrm{L}$. Computerised tomography of brain showed normal sized ventricles and confirmed absence of space occupying lesion or

${ }^{1}$ Senior Lecturer \& Honorary Consultant Paediatric Neurologist, ${ }^{2}$ Probationary Lecturer \& Paediatric Registrar, ${ }^{4}$ Senior Professor of Paediatrics, Faculty of Medicine, University of Colombo, ${ }^{3}$ Consultant Rheumatologist, Lady Ridgeway Hospital, Colombo.

(Received on 17 July 2009. Accepted on 21 August 2009) cerebral oedema. The cellular and biochemical analyses of cerebrospinal fluid (CSF) were normal; culture was sterile. The opening CSF pressure (lateral decubitus position, legs flexed, sedated) was 210 $\mathrm{mmH}_{2} \mathrm{O}$.

A diagnosis of IIH was confirmed. Prolonged use of prednisolone and/or vitamin-A were considered as possible causative agents.

Vitamin A \& D was omitted. After discussion with the rheumatologist, corticosteroid therapy was not withdrawn due to risk of worsening SOJIA. He was given regular pain relief. Treatment with high-dose acetazolamide was commenced and increased up to $50 \mathrm{mg} / \mathrm{kg} /$ day. Frusemide was added, as headache persisted. His symptoms resolved over the next 3-4 weeks. Papilloedema resolved and his visual fields and visual acuity remained unchanged.

\section{Discussion}

"Serous meningitis", "pseudotumour cerebri" and "benign intracranial hypertension" were used to describe increased intracranial pressure in the absence of a space occupying lesion or obstruction to CSF flow. Nosology of "idiopathic intracranial hypertension" (IIH) is preferred, due to the not so benign nature of this condition.

IIH is rare in children. Overall incidence ranges from $0.1-0.9$ per 100,000 population. Incidence is $1-2$ per 100,000 in adults $^{2}$ except obese females (childbearing-age) where it is 19 per 100,000 . Incidence is low in prepubertal years $(<10 \text { years })^{1}$. Higher incidence among adolescents is attributed to increasing obesity among teenagers.

Many drugs and disorders are associated with IIH. The recently implicated associations include diseases and therapies advocated more often in paediatrics. Therapies include recombinant growth factor, desmopressin nasal spray, levenogestral implants and rapid correction of thyroid level with thyroxine in juvenile hypothyroidism. Diseases include acute sinusitis, varicella infection, mumps, Addison disease 
and Guillain-Barre syndrome. Association of IIH and SOJIA is not described but drugs used in its treatment are implicated, making a true association difficult to establish.

Classical symptoms and signs in children include headache $(56-96 \%)$ and papilloedema $(95-100 \%)^{3}$. However, absence of these cardinal features does not exclude $\mathrm{IIH}^{1}$. Another important clinical feature is visual impairment. It occurs as visual field loss, reduction of acuity or blindness ${ }^{4}$. Presentation with classical features is often seen only in older children. Symptoms are different in the very young. An asymptomatic group is also described in children ${ }^{5}$.

Diagnosis of adult IIH is based on modified Dandy's criteria. No criteria are available for IIH diagnosis in children. Most reviews consider $200 \mathrm{mmH}_{2} \mathrm{O}$ to be the upper limit of normal paediatric CSF pressure. Age related cut-offs of $75 \mathrm{~mm}$ in $<2$ years and $135 \mathrm{~mm}$ in $<5$ years are described. A recent review proposed new diagnostic cut-offs for paediatric $\mathrm{IIH}^{1,6}$ dependent on age and presence of papilloedema.

Management includes adequate analgesia, CSF pressure control and ophthalmic surveillance. The treatment guidelines are empirical due to lack of randomized-control-trials in children ${ }^{6}$. Acetazolamide is a first line drug for CSF pressure reduction. Frusemide or corticosteroids are recommended as second line drugs. Surgical interventions are needed for children whose visual loss is deteriorating and /or headache remains intractable despite medical management. These interventions include intermittent CSF drainage, lumbo-peritoneal shunting and opticnerve-sheath fenestration.

A good outcome of paediatric IIH is reported. Most children have complete resolution of papilloedema and visual abnormalities with treatment. However permanent blindness $(0-10 \%)$, visual field loss (17\%) and recurrence $(6-22 \%)$ are reported ${ }^{1}$.

\section{References}

1. Rangwala LM, Liu GT. Paediatric idiopathic intracranial hypertension. Survey of ophthalmology 2007; 52(6): 597-617.

2. Gordon K. Paediatric pseudotumour cerebri: descriptive epidemiology. Canadian Journal of Neurological Sciences 1997; 24: 219-21.

3. Salman MS, Kirkham FJ, MacGregor DL. Idiopathic "benign" intracranial hypertension: Case series and review. Journal of Child Neurology 2001; 16: 465-70.

4. Acheson JF. Idiopathic intracranial hypertension and visual function. [Review] [42refs].British Medical Bulletin 2006; 79-80: 233-44.

5. Weig SG. Asymptomatic idiopathic hypertension in young children. Journal of Child Neurology 2002; 17: 239-41.

6. Mathews YY. Drugs used in childhood idiopathic or benign intracranial hypertension. [Review] [86 refs]. Archives of Disease in Childhood Education \& Practice 2008; 93(1):19-25. 